\title{
Evaluation of the Shift in Antimicrobial Resistance Due to Extended Spectrum Beta-Lactamase and AmpC Producing Enterobacteriaceae in Hampshire England
}

\author{
Fouch S1*, Mitchell J2, Lwaleed B ${ }^{3}$ and Zinkevich V1 \\ ${ }^{1}$ School of Pharmacy and Biomedical Sciences, University of Portsmouth, UK \\ ${ }^{2}$ School of Biological Sciences, University of Portsmouth, UK \\ ${ }^{3}$ Faculty of Health Sciences, University of Southampton, UK
}

\section{Research Article \\ Volume 1 Issue 1}

Received Date: November 20, 2018

Published Date: December 06, 2018

DOI: $10.23880 /$ aabsc-16000109

*Corresponding author: Dr. Sarah Fouch, University of Portsmouth, School of Pharmacy and Biomedical Science, St Michaels Building, White Swan Road, Portsmouth, P01 2DT, UK, Tel: 02392843559; Email: sarah.fouch@port.ac.uk

\section{Abstract}

The aim of this study was to identify shifts in antimicrobial resistance in the Hampshire region including correlations between patient demographic and antibiotic prescribing to inform safe and effective antimicrobial stewardship. 475 ESBL and AmpC producing bacteria, from various infection sites, were obtained from four hospital laboratories in Hampshire, UK, during 2010 and 2012. All isolates were identified to species level. ESBL production and antimicrobial susceptibility testing was performed using disc diffusion methodology. Multiplex PCR and gel electrophoresis was used to detect the $B l a_{\mathrm{CTX}}, B l a_{\mathrm{TEM}}$ and $B l a_{\mathrm{SHV}}$ resistance determinants. Corresponding patient data included patient age, gender, location, clinical details and previous antibiotic therapy. Patient information revealed mean ages of 60 and 62 for the 2010 and 2012 cohorts respectively, with ages ranging from 3 months to 96. ESBL production was the most prevalent resistance mechanism (65\% in 2010, 79\% in 2012), produced mostly by E.coli (85\% in 2010, 84\% in 2012). While 9 of the 13 antibiotics demonstrated increased resistance, 4 demonstrated a decrease. CTX was the most prevalent resistance determinant (38\% in 2010 and $27 \%$ in 2012), followed by dual expression of CTX \& TEM, TEM, TEM \& SHV, SHV, SHV \& CTX and all three resistance determinants. A significant correlation between patient age and joint expression of TEM \& SHV was observed in 2010, in 2012, patient age significantly correlated with joint CTX and SHV expression. Significant differences could also be determined between resistance determinant type and antimicrobial resistance. This study shows that the incidence of ESBL and AmpC infections and resistance to commonly used antibiotics within Hampshire is increasing both within the hospital and community setting. This emphasises the need for judicious antibiotic prescribing to safeguard this valuable medical commodity.

Keywords: Extended Spectrum Beta-Lactamase; Antibiotic Resistance; Antibiotic Prescribing; Infections 


\section{Introduction}

Since the emergence of extended spectrum betalactamase (ESBL) producing organisms in 1983 [1] their prevalence has continued to rise worldwide contributing significantly to antimicrobial resistance. These plasmid mediated bacterial enzymes provide resistance to early and third generation cephalosporins and monobactams. They can be found in Gram negative organisms especially Enterobacteriaceae [2]. ESBL groups have been classified according to their amino-acid sequences. Those first described were derivatives of the beta-lactamase enzymes TEM-1, TEM-2 and SHV-1; further mutations within these classes have since been identified with more than 160 and 100 variants respectively. Studies indicate the distribution of TEM and SHV ESBL resistance are now worldwide [3]. In addition, a new family of ESBL producing bacteria was identified in 1989 called CTX-M due to the enzyme conferring resistance to cefotaxime instead of ceftazidime. Since its discovery there has been over 65 variants of the CTX-M enzyme identified [3].

Until recently most infections caused by ESBL producing bacteria have been reported as nosocomially related, posing as a risk to hospitalised/Immunocompromised patients [4]. However, further evidence now suggests that ESBL producing organisms may be an emerging threat to the general practitioner or outpatient setting [5]. A study published in 2001 (Israel), analysing the antimicrobial susceptibility patterns of 8338 community urinary pathogens, found that $1 \%$ of the Gram Negative isolates produced ESBL resistance [6]. A further study completed in Spain during 2002 isolated CTX-M ESBL producing E.coli from seven patients suffering urinary tract infections who had never been admitted to hospital [7]. Studies in Sweden during 2008 and 2010 investigated the prevalence of faecal ESBL carriage in patients from the community and within a hospital setting. Results during 2008 illustrated rates of $2.1 \%$ within the primary health care units and $1.8 \%$ within hospitalised patients. In 2010 results identified a further raise of faecal carriage with rates of $3 \%$ within primary health care units and $6.8 \%$ within hospitalised patients [8]. Thus, the evident rise in carriage and isolation of ESBL producing organisms both within hospitalised and community patients are likely to limit treatment options.

Empirical therapy prescribed during the clinical diagnosis is often inadequate resulting in failure or delay in treatment, leading to a rise in mortality rates [9]. Agents illustrating susceptibility to ESBL producing Enterobacteriaceae include cephamycins (cefoxitin and cefotetan); however reluctance to prescribe these therapies is due to the ease in which isolates can incur resistance [10]. Currently carbapenems including imipenem, meropenem and ertapenem are chosen as the drug of choice to treat serious ESBL infections. As the numbers of ESBL infections and use of carbapenems rise, concerns include the potential risk of carbapenem resistant mutants [11]. Although rare, resistance has emerged with carbapenem resistant ESBL producing Enterobacteriaceae reported in a range of settings including a hospital in New York [12] and a tertiary medical centre in Israel [13].

The aim of this two year in-depth study was to evaluate the ESBL profile, including antimicrobial resistance patterns and resistance determinant identification, of isolates collected from four hospitals in the Hampshire region during 2010 and 2012. Evaluation of results would identify shifts in antimicrobial resistance and potential relationships between the patient demographic, antibiotic prescribing and ESBL infection.

\section{Materials and Methods}

\section{Ethics}

Full ethical considerations were given to the collection of bacteria, due to all patient details being anonymised at the point of collection, ethical approval was provided by the University of Portsmouth's Ethical Review Committee.

\section{Patient Demographics}

The following anonymised patient details were recorded: age, gender, clinical details, antibiotic history and physical location and are detailed in Table 2.

\section{Bacterial Isolates}

A total of 475 ESBL and/or AmpC producing bacteria collected from various infection sites, were obtained from four hospital laboratories in Hampshire. Isolates were collected on nutrient agar slopes (Oxoid).

\section{Identification and Antimicrobial Susceptibility Testing}

All isolates were identified to species level using a beta-galactosidase PCR [14], for the identification of E.coli [14] (Table 1) and the API20e system (Biomerieux). ESBL production was detected using the D68C AMPC and ESBL detection method (MAST). Antimicrobial susceptibility testing was performed according to the British Society of Antimicrobial Chemotherapy (BSAC) and European 
Committee on Antimicrobial Susceptibility Testing (EUCAST) guidelines (Gentamicin $10 \mu \mathrm{g} / \mathrm{ml}$, Cefotaxime $30 \mu \mathrm{g} / \mathrm{ml}$, Amoxicillin $10 \mu \mathrm{g} / \mathrm{ml}$, Augmentin $30 \mu \mathrm{g} / \mathrm{ml}$, Cefuroxime $\quad 30 \mu \mathrm{g} / \mathrm{ml}$, Piperacillin-Tazobactam $75 / 10 \mu \mathrm{g} / \mathrm{ml}$, Ciprofloxacin $1 \mu \mathrm{g} / \mathrm{ml}$, Meropenem $10 \mu \mathrm{g} / \mathrm{ml}$, Ertapenem $10 \mu \mathrm{g} / \mathrm{ml}$, Ceftazidime $10 \mu \mathrm{g} / \mathrm{ml}$, Amikacin $30 \mu \mathrm{g} / \mathrm{ml}$, cefepime $30 \mu \mathrm{g} / \mathrm{ml}$ and Tigecycline $15 \mu \mathrm{g} / \mathrm{ml}$ ) [15].

\section{Preparation of Bacterial DNA and PCR Amplification}

DNA was prepared using a boiling technique; a single bacterial colony was suspended in $50 \mu$ l of sterile distilled water and heated at $95^{\circ} \mathrm{C}$ for 3 minutes, then spun, for 5 minutes at $13000 \mathrm{~g}$ at room temperature. The supernatant was removed for PCR analysis. Bla $a_{\mathrm{CTX}}, B l a_{\mathrm{TEM}}$ and $B l a_{\mathrm{SHV}}$ genes were detected using multiplex PCR [16] (Table 1). PCR amplification conditions were as follows: initial denaturation step $95^{\circ} \mathrm{C}$ for 15 minutes; 30 cycles of denaturation at $95^{\circ} \mathrm{C}$ for 30 seconds, annealing at $54^{\circ} \mathrm{C}$ for 30 seconds, extension at $72^{\circ} \mathrm{C}$ for 2 minutes followed by a final extension at $72^{\circ} \mathrm{C}$ for 10 minutes. PCR products were separated on a $1 \%$ agarose gel, stained with red safe stain (Invitrogen) in 1xTAE buffer and visualised using a bench top UV system (Alpha Digi Doc).

\begin{tabular}{|c|c|c|c|}
\hline Primer Name & Sequence 5' to 3' & Tm & Amplicon Size \\
\hline$B l a_{\text {SHv }}$ Forward & ATGCGTTATATTCGCCTGTG & 45 & 747 \\
\hline$B l a_{\text {SHV }}$ Reverse & TGCTTTGTTATTCGGGCCAA & 45 & 747 \\
\hline$B l a_{\text {TEM }}$ Forward & TCGCCGCATACACTATTCTCAGAATGA & 53 & 445 \\
\hline Bla $a_{\text {TEM }}$ Reverse & ACGCTCACCGGCTCCAGATTTAT & 53 & 445 \\
\hline$B l a_{\mathrm{CTX}}$ Forward & ATGTGCAGYACCAGTAARGTKATGGC & 54 & 593 \\
\hline Bla $a_{\mathrm{CTX}}$ Reverse & TGGGTRAARTARGTSACCAGAAYCAGCGG & 54 & 593 \\
\hline Beta-Galactosidase 1 & CTTTGCCTGGTTTCCGGCACCAGAA & 60 & 762 \\
\hline Beta-Galactosidase 2 & AACCACCGCACGATAGAGATTCGGG & 60 & 762 \\
\hline
\end{tabular}

Table 1: Primers used for PCR amplification.

\section{Statistical Analysis}

Statistical significance was assessed using the Spearman correlation, Kruskal-Wallis and Mann-Whitney tests, where appropriate as indicated in the text. Values of $P<0.05$ were deemed statistically significant. All statistical analysis was performed using the software programmes Microsoft Excel and Graphpad Prism version 6.01 for Windows 7.

\section{Results}

\section{Patient Demographic}

The demographic profile of patients included in this study is detailed in Table 2.

\section{Patient Age}

Although samples were obtained from patients ranging in age from 3 months to $>90$ years, our demographic analysis indicates that patients over the age of 50 had a higher incidence of ESBL infections (Table 2).

\section{Patient Gender}

A significantly greater proportion of the patients suffering from ESBL infections were female (Table 2). It was unclear whether this sex-specific effect is a reflection of some inherent vulnerability of female patients or the preponderance of specific types of infections in this gender, hence our further analyses of the site of infection.

\section{Infection Site}

The most prevalent infection site was the urinary tract (UTI), Table 2. Within this patient group a higher proportion of females were observed compared to males, nevertheless this difference was not considered statistically significant $P 0.333$. Other infection sites included wounds, with an equal gender ratio observed in 2010 and a 2:1 ratio of males to females in 2012; systemic infections, from females alone in 2010 and a 1:2 ratio between males and females in 2012; respiratory tract infections, from males alone in both year groups and genital infections from female patients.

\section{Patient Location}

The patient demographic information collected within both year groups revealed that the majority of isolates were collected within the GP setting. The in-patient setting was the second most common patient location, followed by accident and emergency and the intensive care unit (ICU). Within 2010, in addition to the locations 
previously mentioned, isolates were collected from the outpatient setting, the antenatal department and from the ministry of defence (Table 2).

\section{Previous Antimicrobial Therapy}

In order to determine if patients with a history of previous antimicrobial therapy were at an increased risk of ESBL resistance or if an agent outside of the prescribing guidelines had been prescribed, details of patients' previous antibiotic therapy were collected. Within both year sets a high proportion of patients $(70 \%$ of the total for 2010 and $74 \%$ for 2012 ) had not recently received an antibiotic, according to the sample request form received within the clinical laboratory. Further analysis of this patient set revealed that in both year groups, a high percentage of patients (76\% in 2010 and $79 \%$ in 2012) who had not received any antimicrobial therapy presented within the community setting (GP, hospital outpatients or the accident and emergency department). Notably, although not statistically significant (P0.33), the mean age for these patients within each year (55 in 2010 and 59 in 2012) was lower than the mean age for the total years (60 and 62). In contrast a smaller number of patients were reported as not receiving antimicrobial therapy within the inpatient environment $(24 \%$ in 2010 and $21 \%$ in 2012). The mean age, although not statistically significant (P0.33), was higher (71 in 2010 and 66 in 2012) compared to the mean year age listed above. Of those patients who were currently prescribed or had recently received antibiotics, 55\% ( $\mathrm{n}=49),(2010)$ and $50 \%(\mathrm{n}=41),(2012)$ presented in the community setting. Within this group of patients, in 2010, 54\% $(n=26)$ had recently received or were currently prescribed an antibiotic outside of the current prescribing guidelines. Within 2012, this figure had reduced, to $28 \%$ $(\mathrm{n}=11)$. A similar picture could be seen when analysing the information obtained from the inpatient setting (59\% $(\mathrm{n}=29)$ of patients in 2010 and $38 \%(\mathrm{n}=16)$ of patients in 2012).

\begin{tabular}{|c|c|c|}
\hline Criteria & Prevalence 2010 & Prevalence 2012 \\
\hline Patient Details & & $62(+/-1.6$ SEM) \\
\hline Average age of patients & $60(+/-1.6$ SEM) & 3 months -96 \\
\hline Median age (range) & $1-96$ & $77 \%(\mathrm{n}=191)$ \\
\hline Females & $78 \%(\mathrm{n}=179)$ & $23 \%(\mathrm{n}=57)$ \\
\hline Males & $22 \%(\mathrm{n}=50)$ & $77 \%(\mathrm{n}=191)$ \\
\hline Patient Location & & $20 \%(\mathrm{n}=50)$ \\
\hline General Practitioner Surgery & $61 \%(\mathrm{n}=140)$ & $2 \%(\mathrm{n}=5)$ \\
\hline In Patient & $27 \%(\mathrm{n}=62)$ & - \\
\hline Accident and Emergency & $7 \%(\mathrm{n}=16)$ & - \\
\hline Out Patients & $2 \%(\mathrm{n}=5)$ & - \\
\hline Intensive Care Unit & $1 \%(\mathrm{n}=2)$ & $94 \%(\mathrm{n}=234)$ \\
\hline Antenatal Clinic & $1 \%(\mathrm{n}=2)$ & $3 \%(\mathrm{n}=8)$ \\
\hline Ministry of Defence & $1 \%(\mathrm{n}=2)$ & $1 \%(\mathrm{n}=2)$ \\
\hline Site of Infection & & $1 \%(\mathrm{n}=2)$ \\
\hline Urine & $96 \%(\mathrm{n}=218)$ & $1 \%(\mathrm{n}=2)$ \\
\hline Wound & $2 \%(\mathrm{n}=5)$ & \\
\hline Blood Culture & $1 \%(\mathrm{n}=2)$ & $74 \%(\mathrm{n}=184)$ \\
\hline Sputum & $1 \%(\mathrm{n}=2)$ & $26 \%(\mathrm{n}=64)$ \\
\hline Genital Swab & $1 \%(\mathrm{n}=2)$ & $79 \%(\mathrm{n}=155)$ \\
\hline Previous Antibiotic Therapy & & $21 \%(\mathrm{n}=41)$ \\
\hline No Therapy & $70 \%(\mathrm{n}=160)$ & $21 \%(\mathrm{n}=11)$ \\
\hline Previous Therapy & $30 \%(\mathrm{n}=69)$ & $79 \%(\mathrm{n}=41)$ \\
\hline No Therapy - Community Patients & $76 \%(\mathrm{n}=125)$ & $24 \%(\mathrm{n}=40)$ \\
\hline Previous Therapy - Community Patients & $24 \%(\mathrm{n}=15)$ & $76 \%(\mathrm{n}=49)$ \\
\hline Previous Therapy - In Patients & & \\
\hline
\end{tabular}

Table 2: Patient demographic comparison between the two year groups. 


\section{Microbiological Analysis}

Organism Identification: Isolate identification revealed that E.coli was the most prevalent organism producing ESBL resistance in this study. Other bacterial species identified included Klebsiella pneumoniae, Enterobacter cloacae, Klebsiella oxytoca, Proteus mirabilis, Citrobacter freundii, Enterobacter aerogenes, Pantoea sp, Citrobacter braakii, Morganella morganii, and Stenotrophomonas maltophilia (Table 3).

Resistance Mechanism: ESBL production was the most prevalent resistance mechanism expressed (Table 3), AmpC production was prevalent to a lesser extent and 3\% of the isolates within 2010 and $4 \%$ of the isolates within 2012, demonstrated expression of both ESBL and AmpC resistance. A proportion of isolates $(23 \%$ (53) in 2010 and $11 \%$ (24) in 2012) required further analysis to confirm ESBL or AmpC expression. Of these isolates, 85\% $(n=45)$ and $67 \%(n=16)$ exhibited positive DNA analysis for the presence of ESBL resistance determinants. There were no significant differences between the resistance type and hospital site, indicating that there is no association between ESBL or AmpC based infection, for any particular year, and location ( $P>0.05$; Kruskal-Wallis).

There were also no significant differences between the identified resistance type compared to the patient gender $(P>0.05$; Mann Whitney) and patient age $(P>0.05$; Kruskal Wallis).

Comparing the percentage of each resistance type according to the patient age range revealed similarities between age groups 11-20, 21-30, 31-40, 51-60 and 6170 in 2010 and age groups $21-30,31-40,41-50$ and 61-70 in 2012 suggesting that these age groups have an equal risk of ESBL and AmpC producing organisms (Figure 1). While in 2010, a high prevalence of ESBL resistance was detected across all age ranges, significantly lower levels of prevalence for AmpC and dual resistance was detected across all age ranges with the exception of age groups 1120 and 21-30 (Figure 1A). This profile was consistent in isolates from 2012 (Figure 1B).

\begin{tabular}{|c|c|c|}
\hline Organism Identification & Prevalence 2010 & Prevalence 2012 \\
\hline Escherichia coli & $85 \%(\mathrm{n}=195)$ & $84 \%(\mathrm{n}=208)$ \\
\hline Klebsiella pneumoniae & $6 \%(\mathrm{n}=13)$ & $4 \%(\mathrm{n}=10)$ \\
\hline Enterobacter cloacae & $3 \%(\mathrm{n}=7)$ & $6 \%(\mathrm{n}=15)$ \\
\hline Klebsiella oxytoca & $3 \%(\mathrm{n}=7)$ & 1 isolate \\
\hline Proteus mirabilis & $1 \%(\mathrm{n}=2)$ & $1 \%(\mathrm{n}=4)$ \\
\hline Enterobacter aerogenes & $1 \%(\mathrm{n}=2)$ & $2 \%(\mathrm{n}=4)$ \\
\hline Citrobacter fruendii & 1 isolate & - \\
\hline Pantoea sp & $1 \%(\mathrm{n}=2)$ & 1 isolate \\
\hline Citrobacter braakii & - & $1 \%(\mathrm{n}=2)$ \\
\hline Morganella morganii & & 1 isolate \\
\hline Stenotrophomonas maltophilia & - & $79 \%(\mathrm{n}=196)$ \\
\hline Resistance Mechanism & & $6 \%(\mathrm{n}=15)$ \\
\hline ESBL & $65 \%(\mathrm{n}=148)$ & $4 \%(\mathrm{n}=10)$ \\
\hline AmpC & $9 \%(\mathrm{n}=21)$ & $11 \%(\mathrm{n}=27)$ \\
\hline ESBL and AmpC & $3 \%(\mathrm{n}=7)$ & $23 \%(\mathrm{n}=53)$ \\
\hline Further Analysis to confirm resistance mechanism & & \\
\hline
\end{tabular}

Table 3: Organism and resistance mechanisms prevalence in 2010 and 2012. 


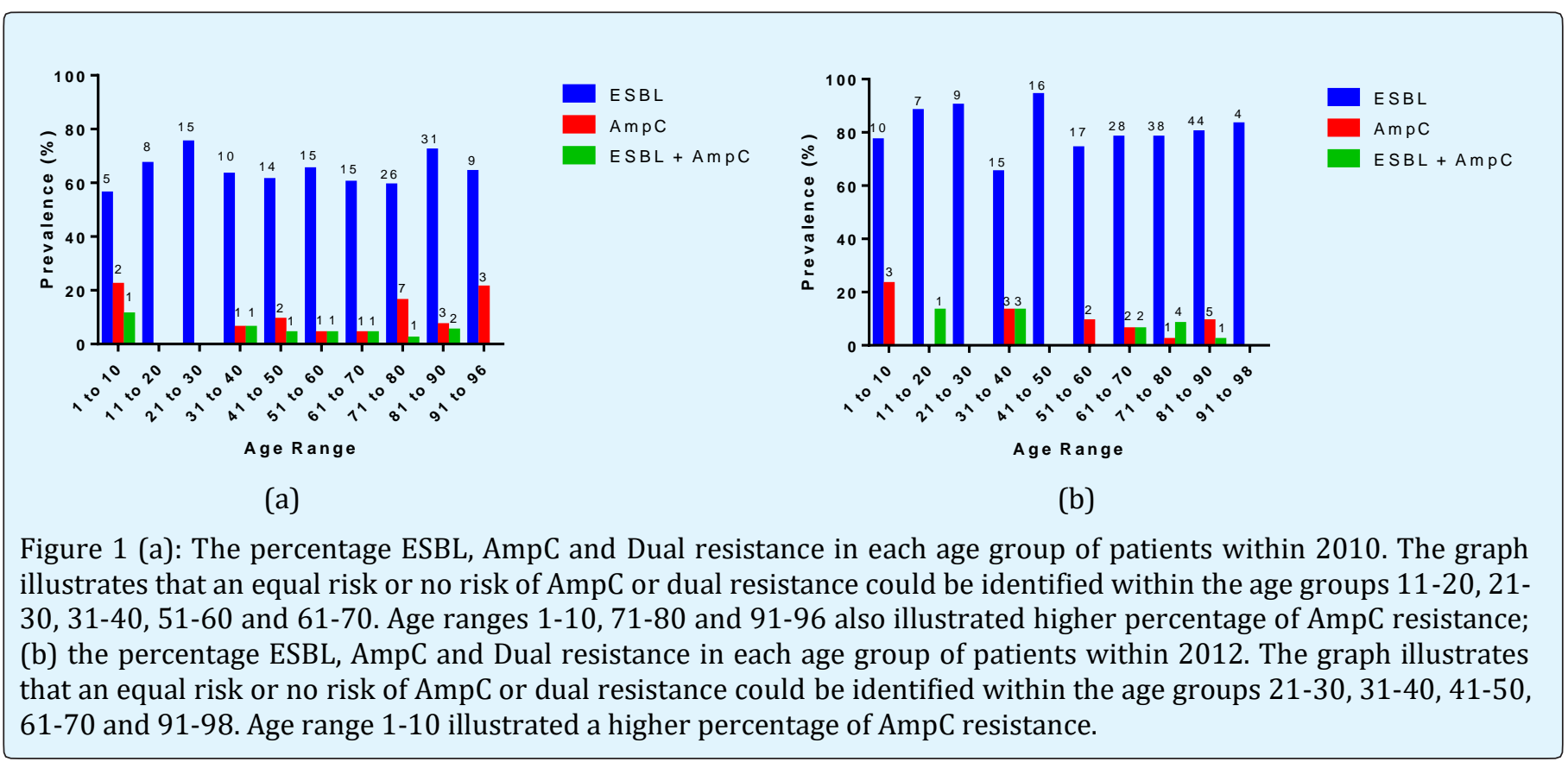

\section{Antimicrobial Susceptibility Investigations}

Antimicrobial susceptibility investigations revealed that amoxicillin exhibited the highest resistance within both year sets; in contrast meropenem demonstrated the highest sensitivity. When comparing the resistance percentages for each year group, 9 of the 13 antibiotics demonstrated increased resistance between 2010 and
2012, these included amoxicillin, augmentin, meropenem, cefotaxime, ertapenem, ceftazidime, tigecycline, cefuroxime and gentamicin. In comparison four of the antibiotics demonstrated a decrease in resistance between the two year groups these included cefepime, ciprofloxacin, piperaillin-tazobactam and amikacin (Figure 2).

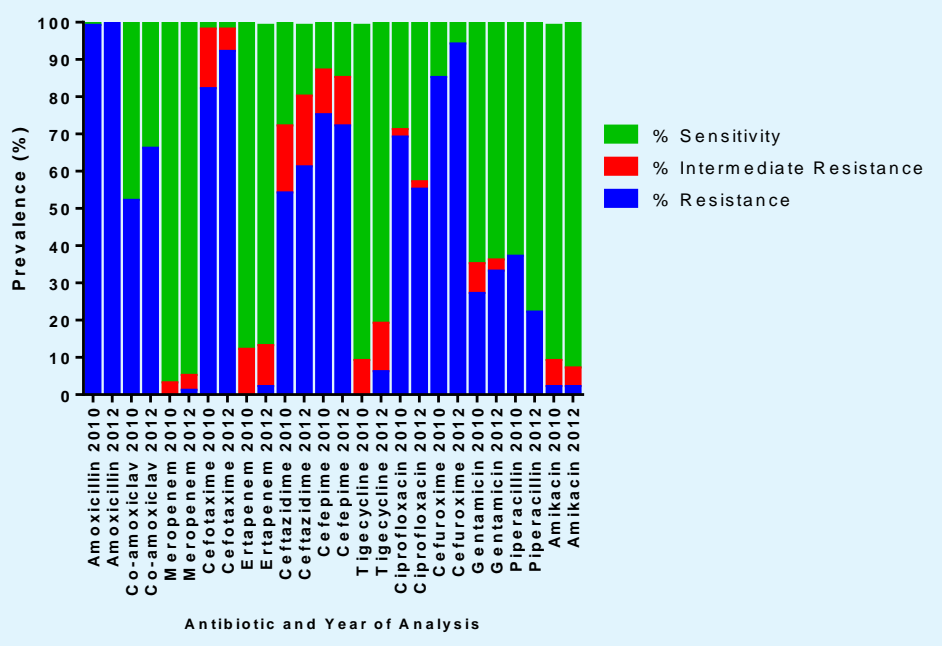

Figure 2: Comparison of percentage resistance, intermediate resistance and sensitivity for each agent within each year group. 
Results illustrate that amoxicillin has the highest and meropenem the lowest resistance rates. An increase in antimicrobial resistance could be identified in amoxicillin, co-amoxiclav, meropenem, cefotaxime, ertapenem, ceftazidime, tigecycline, cefuroxime and gentamicin. In contrast a decrease in antimicrobial resistance could be identified in cefepime, ciprofloxacin, piperaillintazobactam and amikacin.

In order to determine if resistance to specific antimicrobial agents was restricted to or illustrated higher levels within individual hospitals and was perhaps influenced by antimicrobial prescribing policies, each hospital's resistance, intermediate resistance and susceptibility percentages were calculated for each year cohort (Figures 3A and 3B). Comparing the resistance percentages for each hospital, two hospitals demonstrated an increased resistance in augmentin between the years 2010 and 2012 (42\% and 38\% increase). Ertapenem resistance was identified within two hospitals, with a $5 \%$ and $2 \%$ increase between 2010 and 2012 and gentamicin resistance also appeared to increase in three of the hospitals $(7 \%, 10 \%$ and $28 \%$ increases). Tigecycline resistance increased in all of the hospitals, within the two year groups studied $(5 \%, 3 \%, 12 \%$ and $1 \%$ increases). Although the proportions of resistant isolates within each antibiotic category differed for both year cohorts, no significant difference could be determined between the hospital site and resistance to each agent $(\mathrm{P}<0.05$; Kruskal wallis).

\section{Molecular Analysis of Resistance Determinants}

Multiplex PCR and agarose gel electrophoresis allowed for the amplification and separation of three different ESBL resistance determinants, namely TEM, SHV and CTX, (Figure 4). In $20109 \%(\mathrm{n}=20)$ of the isolates and $22 \%$ $(\mathrm{n}=54)$ of the isolates in 2012 expressed TEM alone, compared to $1 \%(n=2,2010)$ and $2 \%(n=4,2012)$ for SHV and $38 \%(n=87,2010)$ and $27 \%(n=66,2012)$ for CTX. Dual expression of CTX and TEM was observed in 34\% $(n=79,2010)$ and $23 \%(n=58,2012)$ of the isolates, $6 \%$ $(n=13,2010)$ and $2 \%(n=5,2012)$ were observed to express TEM and SHV and within 2012 alone $3 \%(n=3)$ of the isolates demonstrated joint resistance with CTX and SHV. One isolate in 2010 and 4 in 2012 expressed all three resistance enzymes and $12 \%(n=27,2010)$ and $21 \%$ $(n=54,2012)$ of the isolates demonstrated negative results for multiplex PCR analysis.

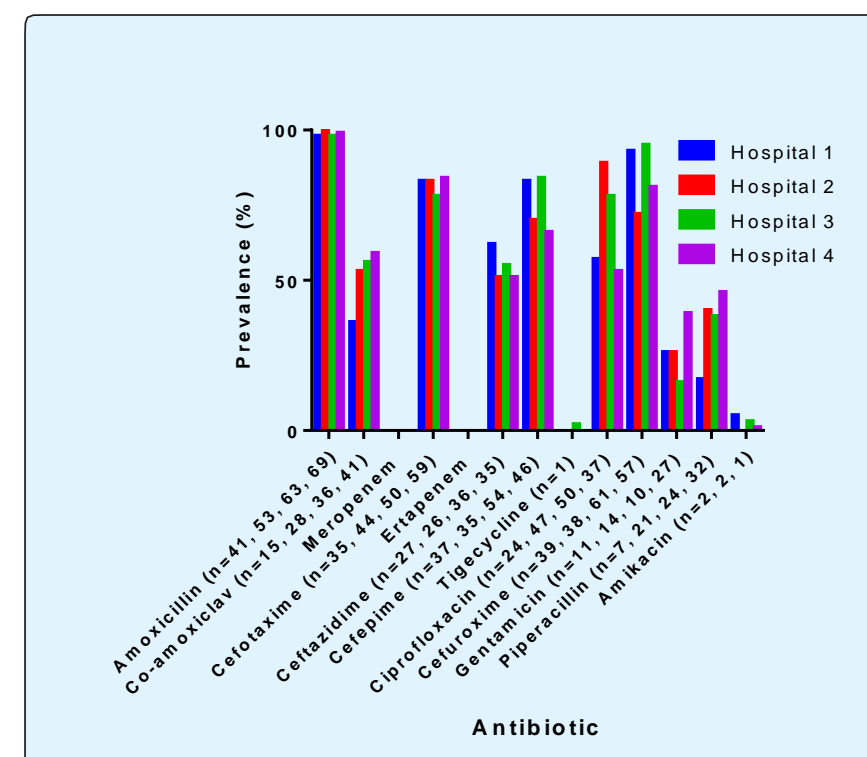

(a)

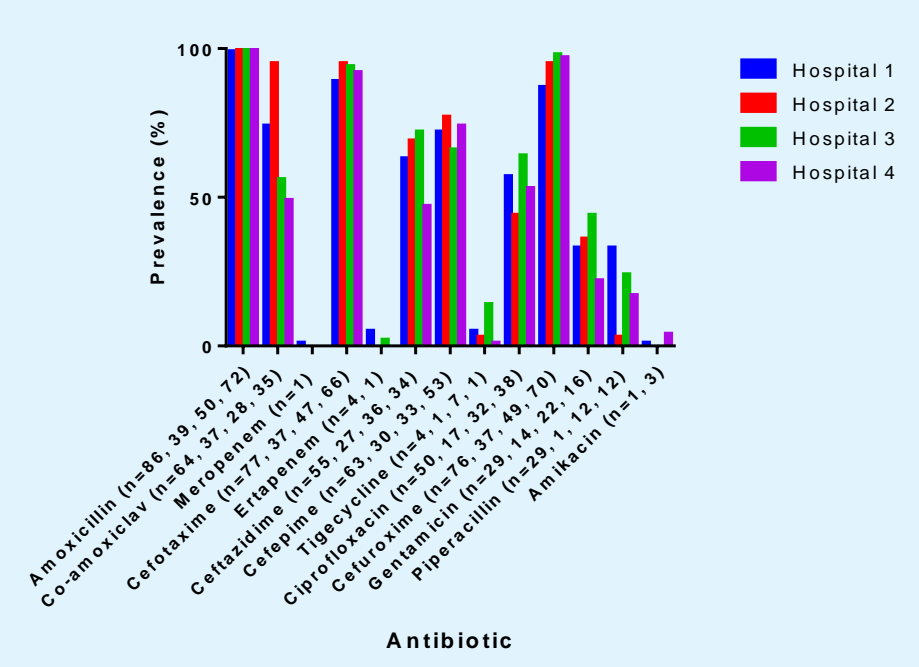

(b)

Figure 3: Percentage resistance against each of the antimicrobial agents tested according to the hospital site within (a) 2010 and (b) 2012. Results indicate that overall resistance is increasing however greater resistance within certain hospitals could be determined within co-amoxiclav, tigecycline, meropenem and ertapenem. 
Lane 123456789101112131415

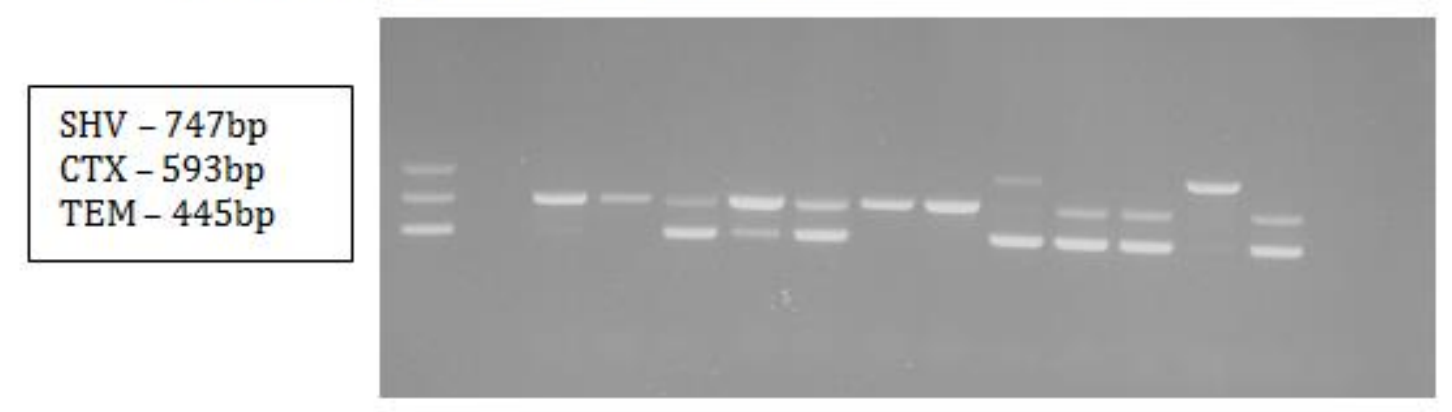

Figure 4: Example of a multiplex PCR analysis for the detection of the SHV, CTX and TEM resistance determinants, lane one illustrates the gene markers, 2-14 sample analysis and lane 15 the negative control.

Although a significant proportion of the isolates were responsible for infections in female patients, no significant differences could be determined between resistance determinant and patient gender or hospital site $(P>0.05$; Mann-Whitney). In the 2010 cohort a relationship between patient age and joint expression of the TEM and SHV determinants was revealed by a Spearman correlation $(P 0.0174)$. The same correlation was not evident in 2012, as resistance had spread to age groups 0-10 and 30-40. In 2012 a similar relationship was observed between patient age and joint CTX and SHV expression (Spearman correlation, p0.0221).

\section{Discussion}

This study aimed to evaluate the profile of ESBL producing bacteria in the Hampshire county and identify relationships between ESBL, AmpC or dual resistance, the patient demographic and antimicrobial prescribing, to highlight potential shifts in antimicrobial resistance. The results obtained have identified some significant findings including an increase in antimicrobial resistance in nine of the thirteen antibiotics studied and potential relationships between resistance determinant and patient age.

The organism identification and patient demographic within the Hampshire area corresponded to previous studies. Results compare to a number of published data including those by Kassakian, et al. who described mean patient ages of 69, 70 and 65, with E.coli presenting as the most common bacterial species and urinary tract infections being the most predominant infection type [17].

The preponderance of isolates collected from female patients suggested an increase susceptibility to multiresistant infection. Nevertheless the predominant infection site in both year cohorts was the urinary tract and, with evidence suggesting that half the female population will suffer a UTI during their lifetime, this could be testament to why a higher number of female infections were observed [18].

A significant number of patients within each year cohort reported as receiving no previous antimicrobial therapy. Further scrutiny of this information revealed that patients presenting within the community had a lower mean age compared to the total age. This implies that these patients fall within younger age groups, with potentially lower risk of predisposing illness prior to experiencing an ESBL infection. While previous antimicrobial therapy has been identified as a risk factor for ESBL production, this information suggests that a significant proportion of patients within this county do not fall within this patient demographic. In contrast patients presenting within the nosocomial environment demonstrated higher age groups compared to the total mean age, implying an increased risk of predisposing pathologies and potential immuno-compromisation resulting in an increased risk of multi-resistant infection.

An increase in prevalence of ESBL infections within the community setting was identified. This result was supported by previous research illustrating that ESBL resistance is not only a concern in the hospital 
environment but within the community setting. No statistically significant relationships could be identified between age and resistance mechanisms, nevertheless further analysis revealed that within both year cohorts patients aged 1-10 appeared to have increased prevalence of AmpC infections. Further research should be initiated to identify potential risk factors, including intestinal carriage, with an aim to reduce AmpC infections within this age group. The most striking observation was the increase in prevalence of ESBL infections across all age ranges, implying that ESBL infections are increasing each year within the Hampshire County.

Antibiotic susceptibility investigations identified an increase in resistance in nine of the thirteen antibiotics studied, of these amoxicillin demonstrated the highest levels of resistance and meropenem the least. Amoxicillin has been widely utilised for the treatment of UTI's, potentially increasing resistance. The National Institute for Health and Care Excellence (NICE) guidelines now only recommend the use of amoxicillin for the treatment of asymptomatic bacteriuria in pregnancy [19]. In contrast meropenem is reserved for patients suffering sepsis and, in comparison is used to a lesser extent.

Information from the primary care trust prescribing data for Hampshire was collected to evaluate antimicrobial resistance to prescribing quantities. A significant increase in antimicrobial prescriptions was identified between the first quarter of 2010-2011 and the fourth quarter of 2011-2012 $(186,465$ to 325,909$)$. Hospital prescribing policies were also scrutinised to distinguish if prescribing in the nosocomial environment could potentially contributed to increases in resistance. Agents demonstrating increased resistance such as augmentin and gentamicin were listed in two or more policies. In contrast ertapenem, identified as an agent with a reduction in resistance was only listed within one hospital prescribing policy. Applying this theory to the increased resistance of tigecycline was challenging as this agent was, at the time, seldom used within the Hampshire county. Previous research nevertheless has identified spontaneous mutations resulting in reduced susceptibility to tigecycline within E.coli and Klebsiella pneumoniae isolates $[20,21]$. Prescribing of individual agents within the community was also investigated through the prescribing by GP practice database, held by the Health and Social Care Information Centre. During the month of June 2010, 2636 doses of augmentin were prescribed, this increased to 2953 in 2012, implying that an increase in prescribing may contribute to antimicrobial resistance. In contrast prescriptions for ciprofloxacin, an agent demonstrating a reduction in resistance, rose by only 112 between 2010 and 2012 (1578 to 1690). Although an increase in prescriptions was identified, the number prescribed was significantly lower than those of augmentin, suggesting that ciprofloxacin is used to a lesser extent. These observations reinforce the importance of the regular review of antimicrobial prescribing policies. Regularly rotating agents may plausibly aid in the reduction of antimicrobial resistance.

A significant correlation was identified between patient age and TEM and SHV expression in the 2010 cohort and between CTX and SHV in 2012. These findings suggest that resistance determinants may initially present within the elderly population and in time increase to a more diverse age range.

To conclude, this study has identified an increase in ESBL prevalence in the nosocomial and community settings. An increased prevalence of AmpC was identified in younger patients and resistance determinant expression potentially emerged in the elderly population, then diversified through all ages. An increase in antibiotic resistance escalates the importance of timely diagnosis and prompt appropriate treatment. Over prescribing of agents may contribute to increased resistance. Action should be taken with regards to antimicrobial stewardship to reduce prescriptions for unnecessary cases, potentially reducing resistance.

\section{References}

1. Coque TM, Baquero F, Canton R (2008) Increasing prevalence of ESBL-producing Enterobacteriaceae in Europe. Euro Surveilliance 13(47): 19044.

2. Pfaller MA, Segreti J (2006) Overview of the epidemiological profile and laboratory detection of extended-spectrum beta-lactamases. Clinical Infectious Diseases 42(S4): S153-S163.

3. Canton R, Novais A, Valverde A, Machado E, Peixe L, et al. (2008) Prevalence and spread of extendedspectrum beta-lactamase-producing Enterobacteriaceae in Europe. Clinical Microbiology and Infection 14(S1): 144-153.

4. Bradford PA (2001) Extended-spectrum betalactamases in the 21st century: characterization, epidemiology, and detection of this important resistance threat. Clinical Microbiology Reviews 14(4): 933-951. 
5. Pitout JD, Nordmann P, Laupland KB, Poirel L (2005) Emergence of Enterobacteriaceae producing extended-spectrum beta-lactamases (ESBLs) in the community. Journal of Antimicrobial Chemotherapy 56(1): 52-59.

6. Colodner R, Keness Y, Chazan B, Raz R (2001) Antimicrobial susceptibility of community-acquired uropathogens in northern Israel. International Journal Antimicrobial Agents 18(2): 189-192.

7. Bou G, Cartelle M, Tomas M, Delia Canle, Francisca Molina, et al. (2002) Identification and broad dissemination of the CTX-M-14 beta-lactamase in different Escherichia coli strains in the northwest area of Spain. Journal of Clinical Microbiology 40(11): 4030-4036.

8. Stromdahl H, Tham J, Melander E, Walder M, Edquist PJ, et al. (2011) Prevalence of faecal ESBL carriage in the community and in a hospital setting in a county of Southern Sweden. European Journal of Clinical Microbiolgy Infectious Disease 30(10): 1159-1162.

9. Pitout JD, Laupland KB (2008) Extended-spectrum beta-lactamase-producing Enterobacteriaceae: an emerging public-health concern. Lancet Infectious Diseases 8(3): 159-166.

10. Pangon B, Bizet C, Bure A, Pichon F, Philippon A, et al. (1989) In vivo selection of a cephamycin-resistant, porin-deficient mutant of Klebsiella pneumoniae producing a TEM-3 beta-lactamase. Journal of Infectious Diseases 159(5): 1005-1006.

11. del Mar Tomas M, Cartelle M, Pertega S, Beceiro A, Llinares P, et al. (2005) Hospital outbreak caused by a carbapenem-resistant strain of Acinetobacter baumannii: patient prognosis and risk-factors for colonisation and infection. Clinical Microbiology Infection 11(7): 540-546.

12. Bradford PA, Bratu S, Urban C, Visalli M, Mariano N, et al. (2004) Emergence of carbapenem-resistant Klebsiella species possessing the class A carbapenemhydrolyzing KPC-2 and inhibitor-resistant TEM-30 beta-lactamases in New York City. Clinical Infectious Diseases 39(1): 55-60.

13. Samra Z, Ofir O, Lishtzinsky Y, Madar-Shapiro L, Bishara J (2007) Outbreak of carbapenem-resistant Klebsiella pneumoniae producing KPC-3 in a tertiary medical centre in Israel. International Journal of Antimicrobial Agents 30(6): 525-529.

14. Pearce CB, Zinkevich V, Beech I, Funjika V, Ruiz AG, et al. (2005) Using the polymerase chain reaction coupled with denaturing gradient gel electrophoresis to investigate the association between bacterial translocation and systemic inflammatory response syndrome in predicted acute severe pancreatitis. World J Gastroenterol 11(45): 7142-7147.

15. Chemotherapy BSoA (2012) Methods for Susceptibility Testing.

16. Monstein HJ, Ostholm-Balkhed A, Nilsson MV, Nilsson M, Dornbusch K, et al. (2007) Multiplex PCR amplification assay for the detection of blaSHV, blaTEM and blaCTX-M genes in Enterobacteriaceae. APMIS 115(12): 1400-1408.

17. Kassakian SZ, Mermel LA (2014) Changing epidemiology of infections due to extended spectrum beta-lactamase producing bacteria. Antimicrobial resistance and infection control 3(1): 9.

18. Foxman B (2003) Epidemiology of urinary tract infections: incidence, morbidity, and economic costs. Dis Mon 49(2): 53-70.

19. National Institute for Health and Care Excellence (2014) Asymptomatic Bacteriuria in Pregnancy.

20. Linkevicius M, Sandegren L, Andersson DI (2013) Mechanisms and fitness costs of tigecycline resistance in Escherichia coli. Journal of Antimicrobial Chemotherapy 68(12): 2809-2819.

21. Sheng ZK, Hu F, Wang W, Qinglan G, Zhijun C, et al. (2014) Mechanisms of Tigecycline Resistance among Klebsiella pneumoniae Clinical Isolates. Antimicrob Agents Chemother 58(11): 6982-6985. 